\title{
Explicit incorporation of asymptotic states into hypoplasticity
}

\author{
D. Mašín \\ Faculty of Science, Charles University in Prague, Czech Republic \\ e-mail: masin@natur.cuni.cz
}

Summary. A rearrangement of the hypoplastic constitutive equation is proposed that enables the incorporation of an asymptotic state boundary surface of an arbitrary pre-defined shape into the model, with any corresponding asymptotic strain rate direction. This opens the way for further development of hypoplastic models. Predictions of a new clay hypoplastic model based on the propsoed approach are shown for demonstration.

Keywords: hypoplasticity, asymptotic behaviour, clay, state boundary surface.

\section{Introduction}

Over the past two decades, hypoplasticity has proven to be a powerful approach to predict the nonlinear behaviour of soils. Early hypoplastic models were developer from sound physical assumptions by means of trial-and-error procedures (see Kolymbas [6]). These models represented the soil behaviour reasonably well, but their parameters did not have any clear physical meaning. Throughout the development of hypoplasticity, several milestones can be identified, and these are often related to predictions of asymptotic states (see Gudehus and Mašín [3], Mašín [9]). Following the earlier work by Gudehus [1] and Niemunis [13], Mašín and Herle [12] analysed asymptotic response of the clay hypoplastic model from Mašín [8]. They found that the predicted asymptotic states depended on the material parameters, while only isotropic and critical states were pre-defined. The complete shape of the asymptotic state boundary surface (ASBS) thus could not be prescribed a priori, as is normal in elasto-plastic models. This property of hypoplasticity has been regarded as its serious limitation.

A procedure for explicit incorporation of any pre-defined asymptotic states into hypoplasticity has recently been developed by Mašín [11], and adopted in a development of a particular clay hypoplastic model in Mašín [10]. Primary outcomes of this research are presented in this contribution. 


\section{Explicit incorporation of asymptotic states into hypoplasticity}

A procedure for explicit incorporation of the asymptotic states into hypoplasticity has been proposed by Mašín [11], and we will adopt this approach herein. A general formulation of the hypoplastic model may be written as [1]

$$
\stackrel{\circ}{\mathbf{T}}=f_{s}\left(\mathscr{L}: \mathbf{D}+f_{d} \mathbf{N}\|\mathbf{D}\|\right)
$$

where $\mathbf{T}$ and $\mathbf{D}$ represent the objective (Zaremba-Jaumann) stress rate and the Euler stretching tensor respectively, $\mathscr{L}$ and $\mathbf{N}$ are fourth- and second-order constitutive tensors, $f_{s}$ is the factor controlling the influence of mean stress (barotropy factor) and $f_{d}$ is the factor controlling the influence of relative density (pyknotropy factor).

To evaluate the model response at the ASBS, we will interpret it in the stress space normalised by the size of the constant void ratio cross-section through the ASBS. It is given by the Hvorslev equivalent pressure $p_{e}$, defined as a mean stress at the isotropic normal compression line at the current void ratio $e$. The normalised stress thus reads $\mathbf{T}_{n}=\mathbf{T} / p_{e}$ and it follows [10] that

$$
\stackrel{\circ}{\mathbf{T}}_{n}=\frac{\stackrel{\circ}{p_{e}}}{p_{e}}-\frac{\mathbf{T}}{p_{e}^{2}} \dot{p}_{e}
$$

In the following, we assume normal compression lines linear in the $\ln (1+e)$ vs. $\ln p / p_{r}$ plane $\left(p_{r}\right.$ is the reference stress of $\left.1 \mathrm{kPa}\right)$. The isotropic normal compression line can be written as

$$
\ln (1+e)=N-\lambda^{*} \ln \left(p_{e} / p_{r}\right)
$$

where $N$ and $\lambda^{*}$ are model parameters. It follows that

$$
p_{e}=p_{r} \exp \left[\frac{N-\ln (1+e)}{\lambda^{*}}\right]
$$

and thus

$$
\dot{p}_{e}=-\frac{p_{e}}{\lambda^{*}}\left(\frac{\dot{e}}{1+e}\right)=-\frac{p_{e}}{\lambda^{*}} \operatorname{tr} \mathbf{D}
$$

Combination of (5), (2) and (1) implies that

$$
\stackrel{\circ}{\mathbf{T}}_{n}=\frac{f_{s}}{p_{e}}\left(\mathscr{L}: \mathbf{D}+f_{d} \mathbf{N}\|\mathbf{D}\|\right)+\frac{\mathbf{T}}{p_{e} \lambda^{*}} \operatorname{tr} \mathbf{D}
$$

During asymptotic stretching the stress state remains fixed in the $\mathbf{T}_{n}$ space [12], provided the constant void ratio cross-sections through the ASBS differ only in size and not in shape. This condition implies $\stackrel{\circ}{\mathbf{T}}_{n}=0$. It then follows from (6) that

$$
-\frac{\mathbf{T}}{\lambda^{*}} \operatorname{tr} \mathbf{D}^{A}=f_{s}\left(\mathscr{L}: \mathbf{D}^{A}+f_{d}^{A} \mathbf{N}\left\|\mathbf{D}^{A}\right\|\right)
$$


where $f_{d}^{A}$ is the value of $f_{d}$ at the ASBS and $\mathbf{D}^{A}$ is the asymptotic strain rate corresponding to the given stress state. Eq. (7) can be manipulated in the following way:

$$
\begin{array}{r}
-\left(\frac{\mathbf{T}}{\lambda^{*}} \operatorname{tr} \mathbf{D}^{A}+f_{s} \mathscr{L}: \mathbf{D}^{A}\right)=f_{s} f_{d}^{A} \mathbf{N}\left\|\mathbf{D}^{A}\right\| \\
-\mathscr{A}: \mathbf{D}^{A}=f_{s} f_{d}^{A} \mathbf{N}\left\|\mathbf{D}^{A}\right\| \\
-\mathscr{A}: d=f_{s} f_{d}^{A} \mathbf{N}
\end{array}
$$

where

$$
\begin{aligned}
& \mathscr{A}=f_{s} \mathscr{L}+\frac{\mathbf{T}}{\lambda^{*}} \otimes 1 \\
& d=\frac{\mathbf{D}^{A}}{\left\|\mathbf{D}^{A}\right\|}
\end{aligned}
$$

Eq. (10) implies that

$$
\mathbf{N}=-\frac{\mathscr{A}: d}{f_{s} f_{d}^{A}}
$$

Combining (13) with (1) yields an alternative expression of the hypoplastic model

$$
\stackrel{\circ}{\mathbf{T}}=f_{s} \mathscr{L}: \mathbf{D}-\frac{f_{d}}{f_{d}^{A}} \mathscr{A}: d\|\mathbf{D}\|
$$

An arbitrary shape of the ASBS can be incorporated into hypoplasticity with the aid of Eq. (14), by appropriate specification of the dependence of $f_{d}^{A}$ on the void ratio and stress ratio. The corresponding asymptotic direction of the strain rate is then prescribed by $d$.

\section{Proposed asymptotic states}

A particular shape of the ASBS and the corresponding strain rate direction has been proposed by Mašín [10]. Due to the limitted space, we do not present herein their full mathematical formulations, but instead we describe their main characteristics. The ASBS is characterised by

- Deviatoric (constant mean stress) cross-sections through the ASBS, which correspond to the failure criterion by Matsuoka and Nakai [7].

- Mobilised friction angle $\varphi_{m}$ is equal to the critical state friction angle $\varphi_{c}$ at $p_{e} / p=2$ (where $p_{e}$ is the Hvorslev equivalent pressure). This also implies position of the critical state line in the $\ln p$ vs. $\ln (1+e)$ plane. 
- $\varphi_{m}$ for $p_{e} / p \rightarrow \infty$ limit to $\varphi_{m} \rightarrow 90^{\circ}$. This ensures that the ASBS does not span into the tensile stress region.

- The surface is $C^{1}$ continuous everywhere except the $\mathbf{T}=0$ state.

The proposed shape of the ASBS, whose mathematical formulation is presented in Ref. [10], is in Fig. 1(a) plotted for the axisymmetric stress state in the normalised stress plane $p / p_{e}$ vs. $q / p_{e}$ for different critical state friction angles. Its shape is compared with the shape of the ASBS by the Modified Cam-clay model. The ASBS has the desired properties. In particular, unlike the ASBS of the Modified Camclay model, it is bound by the $\varphi_{m}=90^{\circ}$ state, which means it is bound within the compression stress range. Different 3D views of the ASBS in the principal stress space are show in Fig. 2.

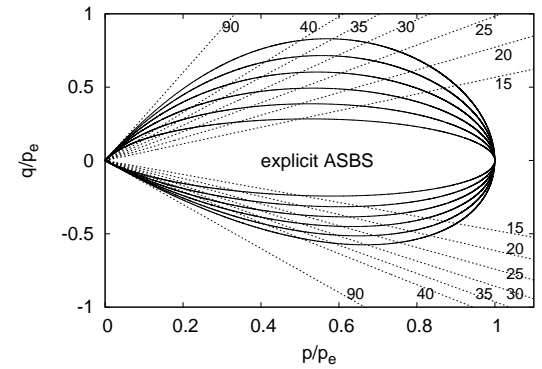

(a)

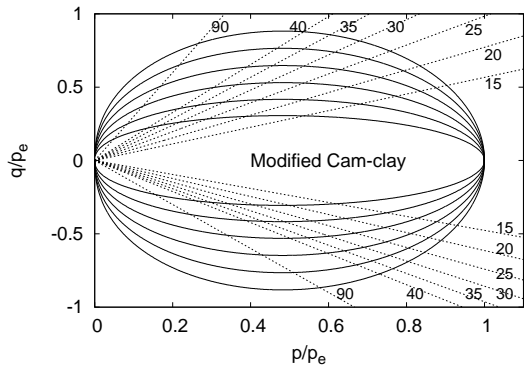

(c)

Fig. 1 Asymptotic state boundary surfaces for different critical state friction angles $\varphi_{c}$. Proposed model (a) and a Modified Cam-clay model (b).

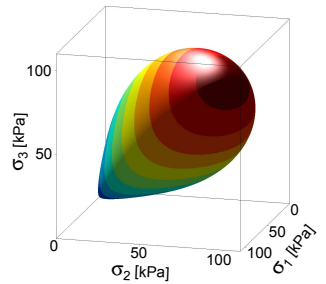

(a)

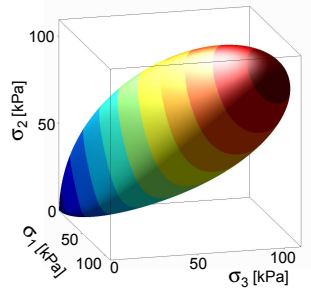

(b)

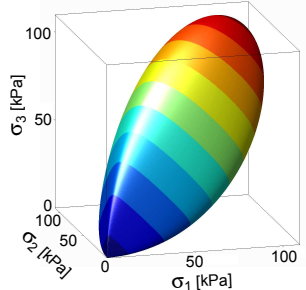

(c)

Fig. 2 Principal stress space 3D plots of the ASBS of the proposed model for $\varphi_{c}=25^{\circ}$.

The proposed asymptotic strain rate direction $d$ has the following properties:

- It predicts zero volumetric strains $(\operatorname{tr} d=0)$ for $\varphi_{m}=\varphi_{c}$.

- It predicts zero shear strains $(\operatorname{dev} d=0)$ for $\varphi_{m}=0^{\circ}$.

- The $K_{0}$ state closely agrees with the Jáky [5] formula 


$$
K_{0}=1-\sin \varphi_{c}
$$

- The $\pm d$ states, introduced by Gudehus [2] (Chapters 2 and 3), are properly considered in both the triaxial compression and extension regime. For details, see $[2,3,9]$.

- $d^{A}$ has radial deviatoric direction.

Figure 3 shows $K_{0}$ values predicted by the proposed [10], original [8] and Modified Cam-clay models for different values of $\varphi_{c}$. The proposed model predicts $K_{0}$ practically coinciding with the Jáky [5] equation (15). Both the original and Camclay models overpredict $K_{0}$.

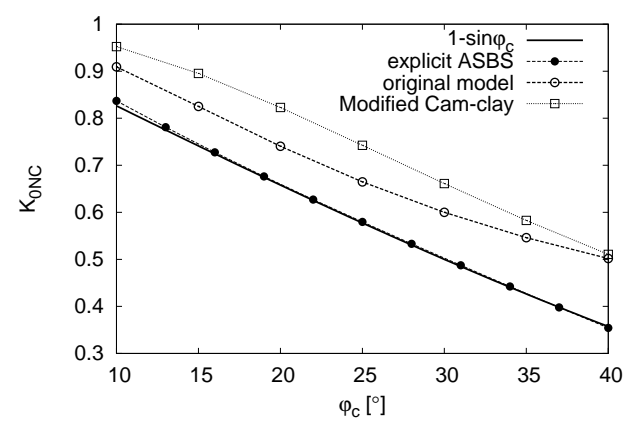

Fig. $3 K_{0}$ values for different $\varphi_{c}$ predicted by the proposed, original and Modified Cam-clay models.

\section{Model formulation}

General rate formulation of the model is given by Eq. (14). The tensor $\mathscr{L}$ is represented by isotropic elasticity: that is

$$
\mathscr{L}=\mathscr{I}+\frac{v}{1-2 v} 1 \otimes 1
$$

where the parameter $v$ controls the proportion of bulk and shear stiffness. Effectively, it regulates the shear stiffness, since the bulk stiffness in the model is controlled by the parameters $\lambda^{*}$ and $\kappa^{*}$ (as shown later). The asymptotic strain rate direction $d$ has been described in Sec. 3. The following expression for the factor $f_{d}$, which governs the non-linear behaviour inside the state boundary surface, is chosen:

$$
f_{d}=\left(\frac{2 p}{p_{e}}\right)^{\alpha}
$$


where $\alpha=2$ is controlling the influence of OCR on non-linear response inside the ASBS. The value of $f_{d}^{A}$ and the asymptotic strain rate direction $d$ is calculated to follow the proposed asymptotic state formulation, described in Sec. 3 .

The last component of the model to be defined is the factor $f_{s}$. It is specified following Ref. [11] to ensure that the slope of the isotropic unloading line in the $\ln (1+e)-\ln p$ plane, for unloading starting from the isotropic normally consolidated state, is given by $\kappa^{*}$. Algebraic manipulations with the above tensorial equations reveal that

$$
f_{s}=\frac{3 p}{2}\left(\frac{1}{\lambda^{*}}+\frac{1}{\kappa^{*}}\right) \frac{1-2 v}{1+v}
$$

The model requires five parameters, with the same physical interpretation as the parameters of the Modified Cam-clay model: $\varphi_{c}, \lambda^{*}, \kappa^{*}, N$ and $v . \varphi_{c}$ is the critical state friction angle; $\lambda^{*}$ is the slope of the isotropic normal compression line in the plane $\ln (1+e)$ vs. $\ln p ; \kappa^{*}$ controls slope of unloading line in the same plane; $N$ is the value of $\ln (1+e)$ at the isotropic normal compression line for $p=p_{r}=1 \mathrm{kPa}$; and finally the parameter $v$ controls the shear stiffness.

\section{Model predictions}

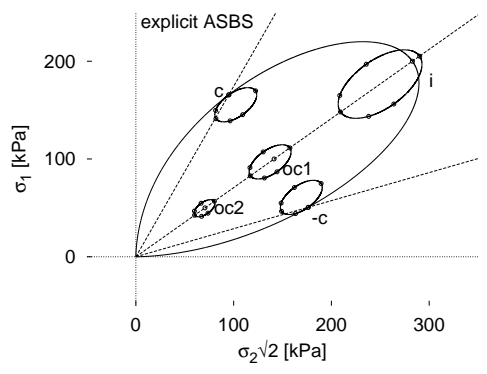

(a)

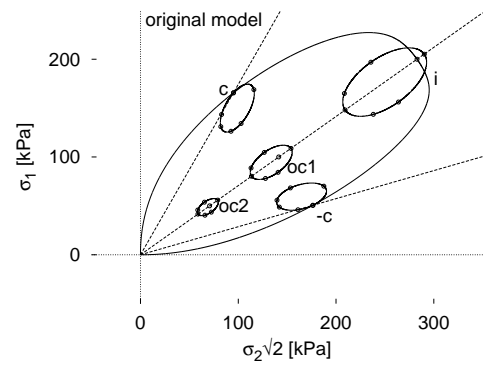

(b)

Fig. 4 The shape of ASBS of the original and proposed models, plotted together with response envelopes at the isotropic $(i)$ and critical $( \pm c)$ asymptotic states and in two overconsolidated (oc) states.

Figure 4 shows the shape of the ASBS, together with response envelopes [3] for different states of stress and overconsolidation. It shows the ASBS predicted by the proposed and original clay hypoplastic models. The original model produces response envelopes which rotate with increasing stress deviator, whereas the proposed model adopts isotropic elasticity, which means that the shape of the response envelopes is not affected by the stress state. This is caused by the different formulation of the tensor $\mathscr{L}$ in the proposed and original models. It influences the model 
predictions inside the ASBS, and causes the proposed model to better reproduce the experimental data.

Figure 5 shows predictions of the undrained triaxial tests on the clay from Dortmund, Germany. The experimental data and predictions by the original model have been presented by Herle et al. [4]. The predicted stress paths differ in the way the asymptotic state is approached, but both predictions represent the experimental data reasonably well. More significant difference is clear from the predicted shear strain vs. deviatoric stress curves. The proposed model predicts accurately the decrease of the tangent shear modulus with shear strain. On the other hand, the original model represents the stress-strain curve relatively poorly, as it underestimates the original stiffness and also the rate of the stiffness degradation.

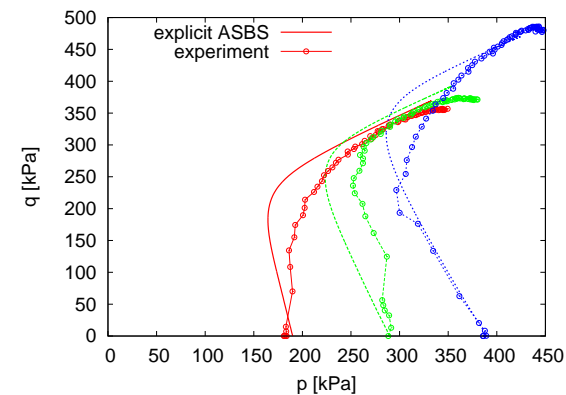

(a)

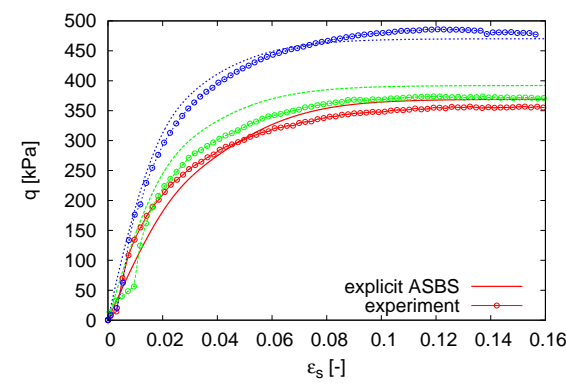

(c)

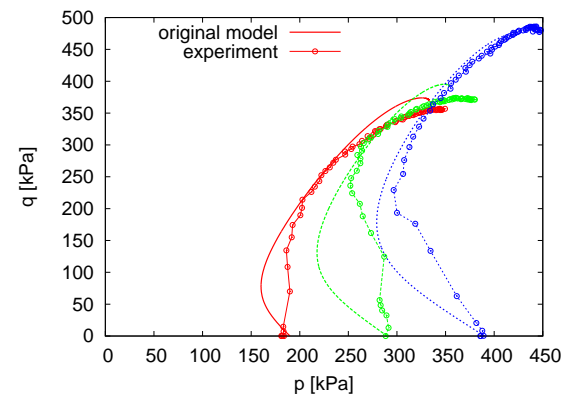

(b)

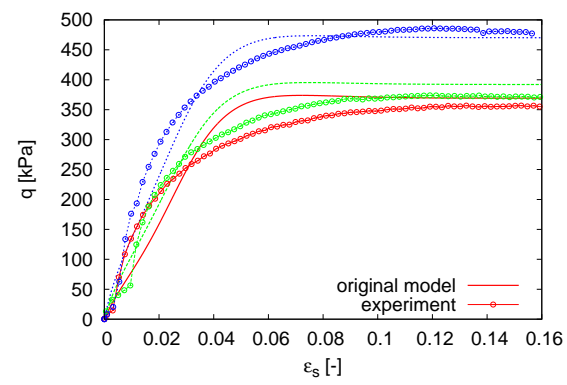

(d)

Fig. 5 Undrained triaxial tests on overconsolidated Dortmund clay and their representation by the proposed and original models. Experimental data from [4].

\section{Conclusions}

A new hypoplastic model for fine-grained soils has been described. The model is based on an approach which enables us to specify explicitly the asymptotic state 
boundary surface and corresponding asymptotic strain rate direction. The model eliminates several shortcomings of the original clay hypoplastic model from [8] and improves its predictions, while using equivalent material parameters. The main advantage of the new model is in the independent formulation of the individual model components. The new model is thus more suitable to form a basis for further developments and enhancements.

Acknowledgements Financial support by the research grants GACR P105/12/1705 and TACR TA01031840 is greatly appreciated.

\section{References}

[1] Gudehus, G.: A comprehensive constitutive equation for granular materials. Soils and Foundations 36(1), 1-12 (1996)

[2] Gudehus, G.: Physical Soil Mechanics. Springer, Berlin (2011)

[3] Gudehus, G., Mašín, D.: Graphical representation of constitutive equations. Géotechnique 59(2), 147-151 (2009)

[4] Herle, I., Mašín, D., Kostkanová, V., Karcher, C., Dahmen, D.: Experimental investigation and theoretical modelling of soft soils from mining deposits. In: Chung, C.K., Jung, Y.H., Kim, H.K., Lee, J.S., Kim, D.S. (eds.) Proc. $5^{\text {th }}$ International Symposium on Deformation Characteristics of Geomaterials, Seoul, Korea. vol. 2, pp. 858-864 (2011)

[5] Jáky, J.: Pressures in silos. In: Proc. $2^{\text {nd }}$ Int. Conf. Soil Mechanics. vol. 1, pp. 103-107. Rotterdam (1948)

[6] Kolymbas, D.: Computer-aided design of constitutive laws. International Journal for Numerical and Analytical Methods in Geomechanics 15, 593-604 (1991)

[7] Matsuoka, H., Nakai, T.: Stress-deformation and strength characteristics of soil under three different principal stresses. In: Proc. Japanese Soc. of Civil Engineers. vol. 232, pp. 59-70 (1974)

[8] Mašín, D.: A hypoplastic constitutive model for clays. International Journal for Numerical and Analytical Methods in Geomechanics 29(4), 311-336 (2005)

[9] Mašín, D.: Asymptotic behaviour of granular materials. Granular Matter (submitted) (2012)

[10] Mašín, D.: Clay hypoplasticity with explicitly defined asymptotic states. Acta Geotechnica (submitted) (2012)

[11] Mašín, D.: Hypoplastic Cam-clay model. Géotechnique 62(6), 549-553 (2012)

[12] Mašín, D., Herle, I.: State boundary surface of a hypoplastic model for clays. Computers and Geotechnics 32(6), 400-410 (2005)

[13] Niemunis, A.: Extended Hypoplastic Models for Soils. Habilitation thesis, Ruhr-University, Bochum (2003) 